\title{
Impact of pulse pressure on clinical outcome in extracorporeal cardiopulmonary resuscitation (eCPR) patients
}

\author{
Jonathan Rilinger ${ }^{1,2}$ (D) Antonia M. Riefler ${ }^{1,2} \cdot$ Xavier Bemtgen $^{1,2} \cdot$ Markus Jäckel $^{1,2} \cdot$ Viviane Zotzmann $^{1,2}$. \\ Paul M. Biever ${ }^{1,2} \cdot$ Daniel Duerschmied ${ }^{1,2} \cdot$ Christoph Benk $^{3} \cdot$ Georg Trummer $^{3} \cdot$ Klaus Kaier $^{4} \cdot$ Christoph Bode $^{1,2}$. \\ Dawid L. Staudacher ${ }^{1,2} \cdot$ Tobias Wengenmayer $^{1,2}$
}

Received: 17 December 2020 / Accepted: 5 March 2021 / Published online: 29 March 2021

(c) The Author(s) 2021

\begin{abstract}
Background Hemodynamic response to successful extracorporeal cardiopulmonary resuscitation (eCPR) is not uniform. Pulse pressure (PP) as a correlate for myocardial damage or recovery from it, might be a valuable tool to estimate the outcome of these patients.

Methods We report retrospective data of a single-centre registry of eCPR patients, treated at the Interdisciplinary Medical Intensive Care Unit at the Medical Centre, University of Freiburg, Germany, between 01/2017 and 01/2020. The association between PP of the first 10 days after eCPR and hospital survival was investigated. Moreover, patients were divided into three groups according to their PP [low $(0-9 \mathrm{mmHg})$, mid $(10-29 \mathrm{mmHg})$ and high $(\geq 30 \mathrm{mmHg})]$ at each time point.

Results One hundred forty-three patients (age 63 years, $74.1 \%$ male, $40 \%$ OHCA, average low flow time 49 min) were analysed. Overall hospital survival rate was $28 \%$. A low PP both early after eCPR (after 1, 3, 6 and $12 \mathrm{~h}$ ) and after day 1 to day 8 was associated with reduced hospital survival. At each time point $(1 \mathrm{~h}$ to day 5$)$ the classification of patients into a low, mid and high PP group was able to categorize the patients for a low (5-20\%), moderate (20-40\%) and high (50-70\%) survival rate. A multivariable analysis showed that the mean PP of the first $24 \mathrm{~h}$ was an independent predictor for survival $(p=0.008)$. Conclusion In this analysis, PP occurred to be a valuable parameter to estimate survival and maybe support clinical decision making in the further course of patients after eCPR.
\end{abstract}

Jonathan Rilinger

jonathan.rilinger@universitaets-herzzentrum.de

1 Department of Medicine III (Interdisciplinary Medical Intensive Care), Medical Center, Faculty of Medicine, University of Freiburg, Freiburg, Germany

2 Department of Cardiology and Angiology I, Faculty of Medicine, Heart Center Freiburg University, University of Freiburg, Hugstetterstr. 55, 79106 Freiburg, Germany

3 Department of Cardiovascular Surgery, Faculty of Medicine, Heart Center Freiburg University, University of Freiburg, Freiburg, Germany

4 Institute of Medical Biometry and Statistics, Faculty of Medicine, University Medical Center Freiburg, University of Freiburg, Freiburg, Germany 


\section{Graphic abstract}

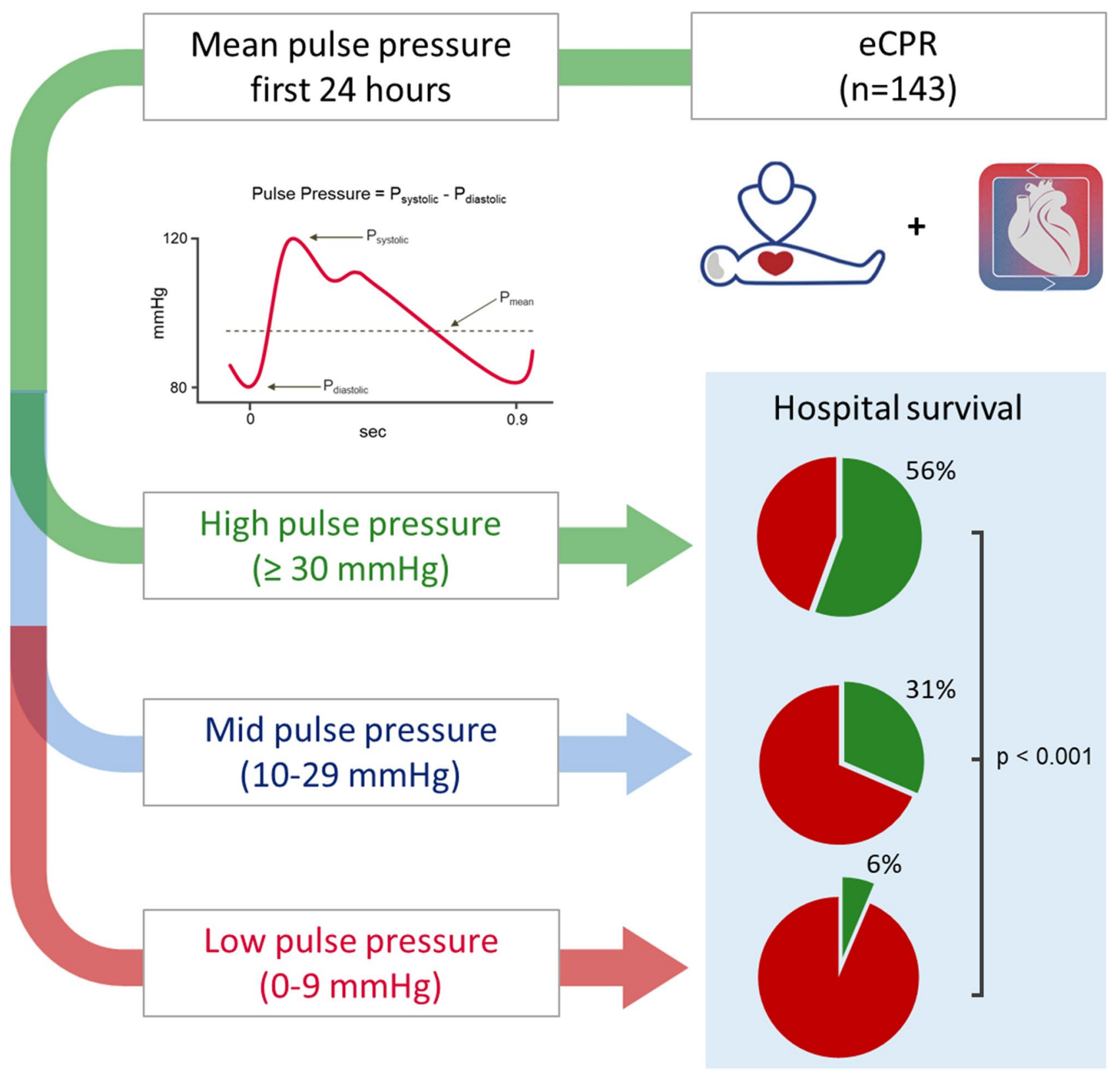

Keywords Pulse pressure $\cdot$ Extracorporeal cardiopulmonary resuscitation $\cdot$ eCPR $\cdot$ Veno-arterial extracorporeal membrane oxygenation $\cdot$ Outcome

Abbreviations

APACHE II score

BMI

BW

CPR

ECMO
Acute physiology and chronic health evaluation

Body mass index

Body weight

Cardiopulmonary resuscitation

Extracorporeal membrane

oxygenation
eCPR

IABP

IHCA

ICU

OHCA

PP

PEA
Extracorporeal cardiopulmonary resuscitation

Intra-aortic balloon pump In hospital cardiac arrest Intensive care unit Out of hospital cardiac arrest Pulse pressure

Pulseless electrical activity 
ROSC

SAPS II score

UHF

VA

Return of spontaneous circulation

Simplified Acute Physiology Score

University Hospital Freiburg

Veno-arterial

\section{Background}

More than 500,000 patients per year suffer cardiac arrest in the US [1] and approximately 275,000 out of hospital cardiac arrests (OHCA) per year are reported for Europe [2]. In case of a refractory cardiac arrest, veno-arterial extracorporeal membrane oxygenation (VA ECMO) support can be considered as a rescue therapy in these patients [3, 4], referred as extracorporeal cardiopulmonary resuscitation (eCPR) $[5,6]$. Both, the increasing availability of VA ECMO systems and the encouraging results of previous studies lead to increasing eCPR numbers [7, 8].

Chances of survival are higher in eCPR patients (28-38\%) [4, 9-12] than in patients treated with conventional cardiopulmonary resuscitation (CPR) (11-14\%) [13, 14]. However, mortality remains high in these very sick patients. Although eCPR represents a promising approach for patients with cardiac arrest, this highly invasive, costly and complex therapy raises many new medical and ethical questions in which effective prognostic assessment plays an important role. So far, superior survival has been demonstrated for an initially defibrillatable heart rhythm, a shorter low flow time as well as a higher $\mathrm{pH}$ and a lower serum lactate level $[15,16]$.

Multiple scores have been established to estimate the possibility of survival after eCPR. Most of these scores rely on parameters from a time point before ECMO initiation [17] and do include the above mentioned parameters.

VA ECMO therapy aims on delivering adequate organ perfusion preventing worsening the post resuscitation syndrome and progression to multi-organ failure. Several biomarkers (e.g. lactate, $\mathrm{pH}$ and standard bicarbonate concentration) reflecting hemodynamic stabilization have been shown to prognosticate survival early on the treatment course [18].

Another evident marker of changes in hemodynamic state is the pulse pressure (PP), reflecting the myocardial function and the level of afterload [19]. In several non-ECMO studies the level of PP was investigated with regards to patient's outcome, obtaining very different results. For instance, a high $\mathrm{PP}$ was associated with lower survival rates in patients with cardiogenic shock in case of an acute coronary syndrome and after myocardial infarction as well as after coronary artery bypasses graft surgery [20-22]. On the other hand, in patients with decompensated heart failure a low PP was a strong predictor for reduced survival [23].

Although it has been shown that PP is able to predict cardiac output in VA ECMO patients [24], so far there is little

evidence on the prognostic value of PP in eCPR patients, especially while ongoing VA ECMO support. Therefore, this retrospective study investigated the influence of $\mathrm{PP}$ on the successful ECMO weaning and hospital survival rate within the first 10 days after eCPR.

\section{Methods}

We report retrospective data of a single-centre registry of patients treated with eCPR. ECPR was defined as VA ECMO implantation during continuous resuscitation or within the first 20 min after return of spontaneous circulation (ROSC) with persistent hemodynamic instability [5]. Decision criteria that support or oppose the use of eCPR in the individual case and the further diagnostic and therapeutic strategy immediately after VA ECMO cannulation were performed in accordance with the German eCPR recommendations [6].

All patients treated at the Interdisciplinary Medical Intensive Care Unit at the Medical Centre, University of Freiburg, Germany, between January 2017 and January 2020 were analysed. Patient identity data derived from the registry were blinded and the study plan was approved by the local ethics committee (EK-Freiburg 151/14). The need for informed consent of the subjects was waived by the local ethics committee.

PP as well as all other analysed parameters were obtained from the medical patient records. Because of diagnostic transfers after VA ECMO implantation (CT scans or coronary angiography) immediately after eCPR, some PP values are missing (additional file 1, Table E7). Patients with missing values at a certain time point were not considered in the survival analysis. For visualization, the course of patients over time in relation to their corresponding pulse pressure PP was extrapolated using the next time-wise available value. After investigating the association between PP and hospital survival, patients were divided into three groups according to their median PP [low (0-9 mmHg), mid (10-29 mmHg) and high $(\geq 30 \mathrm{mmHg})]$.

The primary endpoint was hospital survival. Moreover, successful VA ECMO weaning, 30 day survival and intensive care unit (ICU) survival were analysed. Successful ECMO weaning was defined as free from VA ECMO and alive for at least $48 \mathrm{~h}$ after decannulation. Unsuccessful weaning was defined as the inability to explant the ECMO device because of persistent cardiac failure or death during VA ECMO support or the need for re-cannulation within $48 \mathrm{~h}$. To investigate pulse pressure independently from death due to anoxic brain injury, we also performed a survival analysis excluding these patients.

To compare the patient's disease severity, the SAPS II score [25] at ICU admission was analysed. 
Since, given the same level of diastolic blood pressure, there is collinearity for mean and systolic blood pressure as well as PP, only PP was considered in the multivariable analysis.

\section{Study population}

In this study, only patients with eCPR (VA ECMO cannulation in case of cardiac arrest or instable ROSC) were investigated. Patients with VA ECMO cannulation in case of cardiogenic shock were excluded. All patients admitted to our ICU after eCPR were included in this study. At our institution, patients with in hospital cardiac arrest (IHCA) without ROSC after 15 min were routinely accessed for extracorporeal resuscitation. By local standard, the presence of an unwitnessed cardiac arrest, prolonged duration of CPR without signs of life (breathing, swallowing etc.), a non-shockable initial rhythm and advanced age were considered relative contraindications for VA ECMO cannulation. Final decision to cannulate, however, was driven by a team decision at the bedside including at least two physicians, a perfusionist and two nurses. For OHCA, emergency medical services personal was encouraged to transport patients without ROSC with ongoing chest compressions to the hospital. Since 09/2018 patients with OHCA without ROSC were evaluated for prehospital eCPR. The same relative contra indications applied as for IHCA patients. Cannulation was performed immediately on-site. All patients with evidence of a cardiac cause of the cardiac arrest were primarily assessed by coronary angiography after eCPR and then received a CT scan. Patients with suggestive symptoms or findings of an extra-cardiac cause received a CT scan first. In this case coronary angiography was performed if the CT scan had not provided a sufficient explanation for the cardiac arrest.

\section{ECMO centre and ECMO management}

Our institution features a 24/7 ECMO-centre localized within a tertiary hospital with a 30-bed medical intensive care unit. Cannulations in our ECMO centre are performed by two experienced intensivist and a perfusionist in Seldinger's technique without primary surgical cut down. All members of the ECMO team can be gathered within $30 \mathrm{~min}$. Typical numbers for veno-arterial and veno-venous cannulation are 65 and 35 per year, respectively. There is a $24 \mathrm{~h} / 7$ days outreach team.

Either SCPC (Sorin centrifugal pump console, Livanova, London, United Kingdom) or Cardiohelp systems (Maquet Getinge Group, Rastatt, Germany) were used. Typical venous (draining) cannulas were 21-23 Fr (French = Charrière) in diameter and $55 \mathrm{~cm}$ of length while arterial (returning) cannulas were 15-17 Fr and 15-23 cm (both HLS cannula, Maquet Getinge Group, Rastatt, Germany). Since
January 2019 decannulation is performed under fluoroscopic control using the MANTA Vascular Closure Device ${ }^{\circledR}[26]$, whenever possible.

For patients without life threatening bleeding, anticoagulation was provided by intravenous unfractionated heparin aiming at a partial thromboplastin time 1.5 times upper normal limit.

The management of vasopressors and fluid therapy was driven by clinical judgement of the ECMO experienced intensivist in charge and has been reported earlier [27, 28]. Within the first $48 \mathrm{~h}$ of ECMO support blood flow was kept the highest possible flow without the need for excessive volume substation. All patients received invasive blood pressure monitoring via the right radial artery and cerebral NIRS monitoring.

Moreover, within the first $48 \mathrm{~h}$ of ECMO support no inotropes were applied if there was detectable pulse pressure. Only in case of severe pulmonary edema or if echocardiography showed no opening of the aortic valve inotropes therapy was started. In particularly severe cases, in which despite these measures, severe pulmonary edema or insufficient opening of the aortic valve persisted, mechanical left heart decompression using the Impella ${ }^{\circledR}$ device was performed. The rationale of this algorithm is to avoid additional mechanical support to minimise additional complications. In one case intra-aortic balloon pump (IABP) was placed in addition to the VA ECMO therapy.

Treatment algorithms and standard operating procedures were subject to optimizations during the observational period, reflecting current state of the art recommendations and scientific knowledge.

\section{Statistical analysis}

Continuous variables are presented as median and interquartile range (IQR) and categorical variables as numbers and percentages. Mann-Whitney $U$ test was used for the analysis of continuous variables, Pearson's Chi-squared test or Fisher's exact test for categorical variables. Kruskal-Wallis test was used for the analysis of continuous variables in more than two groups. Multivariable regression analysis was performed for univariate (dependent) predictors of hospital survival (threshold of $p<0.05$ ). Results are given as odds ratio [(OR), 95\% confidence interval $(\mathrm{CI})]$. 60-day survival was calculated using the Kaplan-Meier method. Analyses were exploratory in nature. As a result, there was no prespecified plan to adjust for multiple comparisons and inferences drawn from $95 \%$ confidence intervals or $p$ values may not be reproducible. Statistical calculations were performed using IBM SPSS statistics 25.0 (Armonk, NY: IBM Corp, 2017). Survival analysis was conducted in $R 32$ and figures were produced using the package ggplot 233 . 


\section{Results}

\section{Patients}

Two hundred thirty-two patients were treated with VA ECMO from 01/2017 to 01/2020. After exclusion of 89 patients treated for cardiogenic shock, 143 patients with eCPR (age 63 years, $74.1 \%$ male) could be analysed (Fig. 1). VA ECMO cannulation was performed in 135 patients while CPR and in 8 patients with unstable ROSC.

OHCA occurred in $39.9 \%$ of the patients and in $60.1 \%$ cardiac arrest had a coronary genesis (Table 1). Median no flow and low flow time were $0 \mathrm{~min}$ and $49 \mathrm{~min}$, respectively. In 12 (8.4\%) patients, eCPR was performed prehospital and $42 \%$ of all patients showed a shockable rhythm before VA ECMO implantation (additional file 1, table E1). SAPS II at ICU admission was 56.

Overall successful VA ECMO weaning and hospital survival rate were $35.7 \%$ and $28 \%$, respectively (Table 2). Six $(4.2 \%)$ patients received additional Impella ${ }^{\circledR}$ support and one (0.7\%) patient additional IABP support.

\section{Pulse pressure analysis}

Throughout from 1 h to day 8 after eCPR, a low pulse pressure correlated with reduced hospital survival (additional file 1, table E2). A similar association was observed between low PP and reduced VA ECMO weaning rate (additional file 1, table E3). There was no difference in measured PP pre VA ECMO implantation (while CPR) in survivors and nonsurvivors (only applicable for patients with unstable ROSC).

The classification of patients into a low $(0-9 \mathrm{mmHg})$, mid $(10-29 \mathrm{mmHg})$ and high $(\geq 30 \mathrm{mmHg})$ PP group showed a good discrimination between low, medium and high survival rates at each time point from $1 \mathrm{~h}$ until day 5 after eCPR (Table 3). The same association was shown in the subgroup of patients with ongoing VA ECMO only at each time point (additional file 1, table E4).

Although low or high PP at each single time point showed a strong association to increased mortality or survival rates, many patients switched between the different PP allocations in the first $24 \mathrm{~h}$ (Fig. 2). The Sankey chart visualizes the course of patients over the time in relation to their corresponding pulse pressure. Particularly, a large proportion of patients had alternating low or mid PP in the first $24 \mathrm{~h}$. At each time point a high number of patients presenting with a low PP were dying, while mortality of patients with a high PP was very low. All over, the proportion of deceased patients and patients with a high PP was constantly increasing while the proportion of patients with a low or mid PP was decreasing.

\section{Analysis of mean $24 \mathrm{~h}$ pulse pressure}

There were no differences between the low, mid and high PP groups in sex and BMI, but patients with a low PP were younger (61 vs. 65 and 65 years, $p=0.040$, Table 1). Moreover, patients with a low PP showed a higher rate of OHCA (55\% vs. $35 \%$ and $25 \%, p=0.013$ ) compared to patients with a mid and high PP. The underlying cause of cardiac arrest was similar in all three groups, with $50-60 \%$ of patients showing a coronary cause. Low PP patients showed higher creatine kinase and creatine kinase-MB levels than patients with mid and high $\operatorname{PP}(p=0.015$ and $p=0.018)$.

There were no differences in the no flow time between the three groups, but patients with a low PP showed a
Fig. 1 Study flow chart. Pulse pressure was analysed in 143 patients after eCPR. Patients were allocated to a low-, midand high-pulse pressure group for outcome prediction. ECMO extracorporeal membrane oxygenation, $e C P R$ extracorporeal cardiopulmonary resuscitation, $P P$ pulse pressure and $V A$ venoarterial

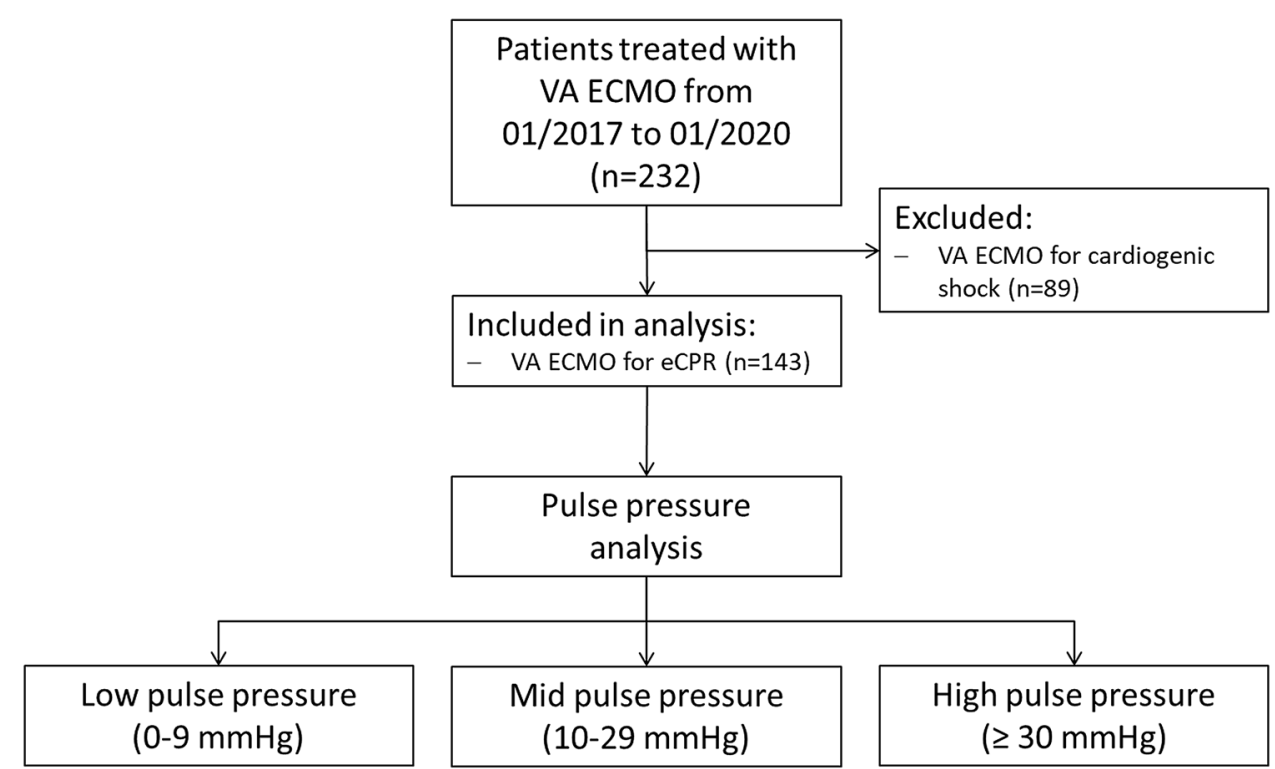


Table 1 Baseline characteristics of the low-, mid- and high-pulse pressure groups

\begin{tabular}{|c|c|c|c|c|c|}
\hline & All patients $(n=143)$ & Low PP $(0-9 \mathrm{mmHg}, n=53)$ & $\begin{array}{l}\text { Mid PP }(10- \\
29 \mathrm{mmHg}, n=54)\end{array}$ & $\begin{array}{l}\text { High PP } \\
(\geq 30 \mathrm{mmHg}, \\
n=36)\end{array}$ & $p$ value \\
\hline Age (years) & $63(54-73)$ & $61(49-70)$ & $65(58-73)$ & $65(57-76)$ & 0.040 \\
\hline Sex (male) & $106(74.1 \%)$ & $40(75.5 \%)$ & $37(68.5 \%)$ & $29(80.6 \%)$ & 0.425 \\
\hline $\mathrm{BMI}\left(\mathrm{kg} / \mathrm{m}^{2}\right)$ & $25(24-28)$ & $24(24-28)$ & $25(24-28)$ & $25(24-28)$ & 0.575 \\
\hline Location of cardiac arrest & & & & & 0.013 \\
\hline IHCA & $86(60.1 \%)$ & $24(45.3 \%)$ & $35(64.8 \%)$ & $27(75 \%)$ & \\
\hline OHCA & $57(39.9 \%)$ & $29(54.7 \%)$ & $19(35.2 \%)$ & $9(25 \%)$ & \\
\hline Cause of cardiac arrest & & & & & 0.341 \\
\hline Coronary & $86(60.1 \%)$ & $35(66 \%)$ & $33(61.1 \%)$ & $18(50 \%)$ & \\
\hline Cardiac, non-coronary & $32(22.4 \%)$ & $10(18.9 \%)$ & $14(25.9 \%)$ & $8(22.2 \%)$ & \\
\hline Other & $25(17.5 \%)$ & $8(15.1 \%)$ & $7(13 \%)$ & $10(27.8 \%)$ & \\
\hline No flow time pre VA ECMO (min) & $0(0-0)$ & $0(0-2.8)$ & $0(0-0)$ & $0(0-0)$ & 0.166 \\
\hline Low flow time pre VA ECMO (min) & $49(30-70)$ & $55(35-75)$ & $44(25-70)$ & $43(29-64)$ & 0.115 \\
\hline SAPS II at ICU admission & $56(48-64)$ & $61(54-68)$ & $53(46-62)$ & $52(46-61)$ & 0.004 \\
\hline Lactate $3 \mathrm{~h}$ after VA ECMO $(\mathrm{mmol} / \mathrm{l})$ & $7.5(4.1-11.6)$ & $7(4.1-11.6)$ & $7.7(2.6-11)$ & $10.3(4.8-17)$ & 0.635 \\
\hline Creatine kinase (U/l) & $589(214-2832)$ & $1107(244-6762)$ & $561(166-1783)$ & $361(177-851)$ & 0.015 \\
\hline Creatine kinase-MB (U/l) & $182(105-525)$ & $273(122-819)$ & $163(120-400)$ & $105(56-258)$ & 0.018 \\
\hline Myoglobin (ng/ml) & $1385(399-4795)$ & $3026(413-9015)$ & $1373(407-3520)$ & $995(337-1936)$ & 0.138 \\
\hline \multicolumn{6}{|l|}{ Comorbidities } \\
\hline Coronary artery disease & $52(36.4 \%)$ & $14(26.4 \%)$ & $19(35.2 \%)$ & $19(52.8 \%)$ & $\mathbf{0 . 0 3 9}$ \\
\hline Chronic heart failure & $22(15.4 \%)$ & $6(11.3 \%)$ & $7(13 \%)$ & $9(25 \%)$ & 0.176 \\
\hline Chronic renal failure & $25(17.5 \%)$ & $6(11.3 \%)$ & $8(14.8 \%)$ & $11(30.6 \%)$ & 0.052 \\
\hline Liver cirrhosis & $12(8.4 \%)$ & $1(1.9 \%)$ & $7(13 \%)$ & $4(11.1 \%)$ & 0.094 \\
\hline Pulmonary diseases & $27(18.9 \%)$ & $10(18.9 \%)$ & $8(14.8 \%)$ & $9(25 \%)$ & 0.481 \\
\hline
\end{tabular}

$p$ values $<0.05$ are presented in bold.

Table shows baseline characteristics of the pulse pressure groups (low, mid and high) for patients alive after $24 \mathrm{~h}$. Mean pulse pressure of the first $24 \mathrm{~h}$ was used for group definition

$B M I$ body mass index, ECMO extracorporeal membrane oxygenation, $I C U$ intensive care unit, $I H C A$ in hospital cardiac arrest, $O H C A$ out of hospital cardiac arrest, PP pulse pressure, SAPS II score Simplified Acute Physiology Score and VA veno-arterial

Table 2 Procedural characteristics and outcome of the low-, mid- and high-pulse pressure groups

\begin{tabular}{|c|c|c|c|c|c|}
\hline & $\begin{array}{l}\text { All patients } \\
(n=143)\end{array}$ & $\begin{array}{l}\text { Low PP } \\
(0-9 \mathrm{mmHg}, n=53)\end{array}$ & $\begin{array}{l}\text { Mid PP }(10- \\
29 \mathrm{mmHg}, n=54)\end{array}$ & $\begin{array}{l}\text { High PP ( } \geq 30 \mathrm{mmHg}, \\
n=36)\end{array}$ & $p$ value \\
\hline Successful ECMO weaning & $51(35.7 \%)$ & $8(15.1 \%)$ & $21(38.9 \%)$ & $22(61.1 \%)$ & $<0.001$ \\
\hline 30 day survival & $44(30.8 \%)$ & $5(9.4 \%)$ & $18(33.3 \%)$ & $21(58.3 \%)$ & $<0.001$ \\
\hline ICU survival & $42(29.4 \%)$ & $4(7.5 \%)$ & $18(33.3 \%)$ & $20(55.6 \%)$ & $<0.001$ \\
\hline Hospital survival & $40(28 \%)$ & $3(5.7 \%)$ & $17(31.5 \%)$ & $20(55.6 \%)$ & $<0.001$ \\
\hline Additional Impella ${ }^{\circledR}$ support & $6(4.2 \%)$ & $4(7.6 \%)$ & $2(3.7 \%)$ & $0(0 \%)$ & 0.134 \\
\hline Additional IABP support & $1(0.7 \%)$ & $0(0 \%)$ & $1(1.8 \%)$ & $0(0 \%)$ & 0.134 \\
\hline Norepinephrine mean 24 h ( $\mu \mathrm{g} / \mathrm{kg}$ BW/min) & $0.4(0.2-0.5)$ & $0.4(0.2-0.7)$ & $0.2(0.1-0.5)$ & $0.3(0.2-0.5)$ & 0.575 \\
\hline Epinephrine mean 24 h ( $\mu \mathrm{g} / \mathrm{kg} \mathrm{BW} / \mathrm{min})$ & $0(0-0.1)$ & $0(0-0.4)$ & $0(0-0.1)$ & $0(0-0.1)$ & 0.642 \\
\hline Dobutamine mean $24 \mathrm{~h}(\mu \mathrm{g} / \mathrm{kg} \mathrm{BW} / \mathrm{min})$ & $0(0-0)$ & $0(0-0)$ & $0(0-0)$ & $0(0-29.2)$ & 0.436 \\
\hline ICU length of stay (days) & $4.8(1.3-12.5)$ & $1.9(0.4-7.6)$ & $4.8(2-11.9)$ & $8.1(5.3-20.4)$ & $<0.001$ \\
\hline VA ECMO duration (days) & $2.8(0.8-4.8)$ & $1.3(0.4-4.3)$ & $3.1(1.6-5.1)$ & $2.9(0.9-4.9)$ & 0.032 \\
\hline
\end{tabular}

$p$ values $<0.05$ are presented in bold.

Mean pulse pressure of the first $24 \mathrm{~h}$ was used for group definition

$B W$ body weight, $E C M O$ extracorporeal membrane oxygenation, $I A B P$ intra-aortic balloon pump, $I C U$ intensive care unit, $P P$ pulse pressure and $V A$ veno-arterial 
Table 3 Survival rates of patients with low-, mid- and high-pulse pressure at different time points

\begin{tabular}{lcccr}
\hline & Low PP (0-9 mmHg) & $\begin{array}{l}\text { Mid PP }(10- \\
29 \mathrm{mmHg})\end{array}$ & High PP $(\geq 30 \mathrm{mmHg})$ & $p$ value \\
\hline PP pre implant & $31(28.4 \%)$ & $0(0 \%)$ & $4(33.3 \%)$ & 0.158 \\
PP 1 h & $1(8.3 \%)$ & $5(21.7 \%)$ & $11(50 \%)$ & $\mathbf{0 . 0 2 2}$ \\
PP 3 h & $9(22.5 \%)$ & $12(24.5 \%)$ & $15(50 \%)$ & $\mathbf{0 . 0 2 4}$ \\
PP 6 h & $8(14 \%)$ & $16(39 \%)$ & $16(55.2 \%)$ & $<\mathbf{0 . 0 0 1}$ \\
PP 12 h & $9(17.6 \%)$ & $16(38.1 \%)$ & $14(56 \%)$ & $\mathbf{0 . 0 0 3}$ \\
PP 24 h & $4(10.3 \%)$ & $19(52.8 \%)$ & $17(51.5 \%)$ & $<\mathbf{0 . 0 0 1}$ \\
PP mean of the first 24 h & $3(6.4 \%)$ & $17(31.5 \%)$ & $20(55.6 \%)$ & $<\mathbf{0 . 0 0 1}$ \\
PP d2 & $1(6.7 \%)$ & $8(26.7 \%)$ & $31(66 \%)$ & $<\mathbf{0 . 0 0 1}$ \\
PP d3 & $0(0 \%)$ & $5(26.3 \%)$ & $35(62.5 \%)$ & $<\mathbf{0 . 0 0 1}$ \\
PP d5 & $0(0 \%)$ & $4(28.6 \%)$ & $36(66.7 \%)$ & $\mathbf{0 . 0 0 9}$ \\
PP d7 & $0(0 \%)$ & $2(40 \%)$ & $35(71.4 \%)$ & 0.127 \\
\hline
\end{tabular}

$p$ values $<0.05$ are presented in bold.

Hospital survival rates are shown pre implant and for advancing time periods after eCPR for the three pulse pressure groups

$e C P R$ extracorporeal cardiopulmonary resuscitation and $P P$ pulse pressure

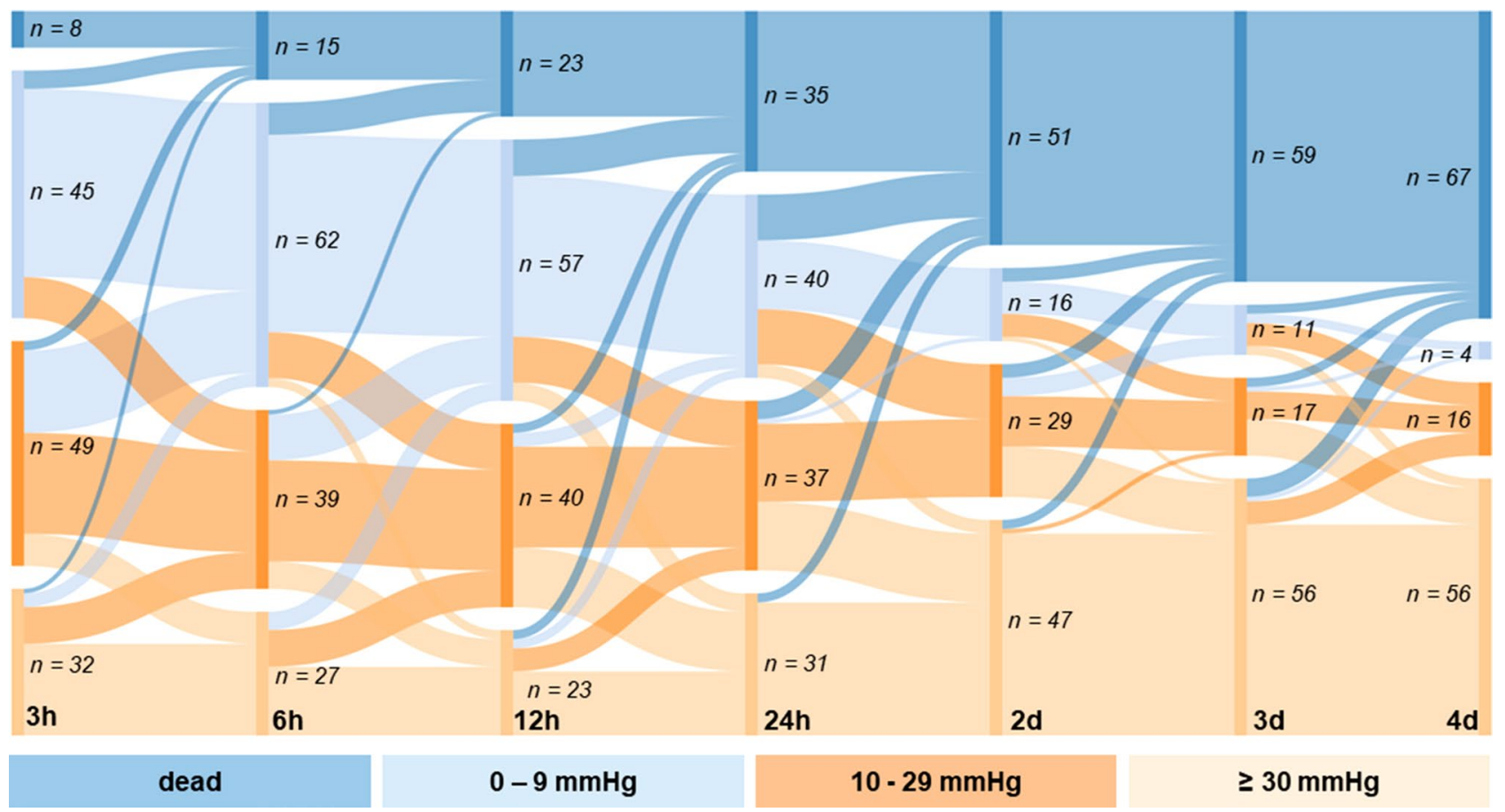

Fig. 2 Sankey chart displaying the course of patients over the time in relation to their corresponding pulse pressure. $e C P R$ extracorporeal cardiopulmonary resuscitation and $P P$ pulse pressure

numerically but not statistically significant higher low flow time (55 min vs. $44 \mathrm{~min}$ and $43 \mathrm{~min}$ ). Moreover, SAPS II at hospital admission was higher in the low PP group. Interestingly, the rate of underlying coronary artery disease was higher in the mid and high PP group $(p=0.039)$. Furthermore, there was a trend for a higher rate of chronic renal failure in patients with a high $\mathrm{PP}(p=0.052)$.
Successful VA ECMO weaning rate was higher for patients with a high PP (61\%) than for patients with mid (39\%) and low PP $(15 \%, p<0.001$, Table 2). Hospital survival rates for patients with a mean low, mid and high $\mathrm{PP}$ in the first $24 \mathrm{~h}$ after eCPR were $5.7 \%, 31.5 \%$ and $55.6 \%$, respectively $(p<0.001$, Fig. 3). After exclusion of patients died due to anoxic brain injury or withdrawal of care in those circumstances, survival rates were $7.9 \%, 42.5 \%$ and $71.4 \%$, respectively $(p<0.001)$. 
+ Low pulse pressure + Mid pulse pressure + High pulse pressure

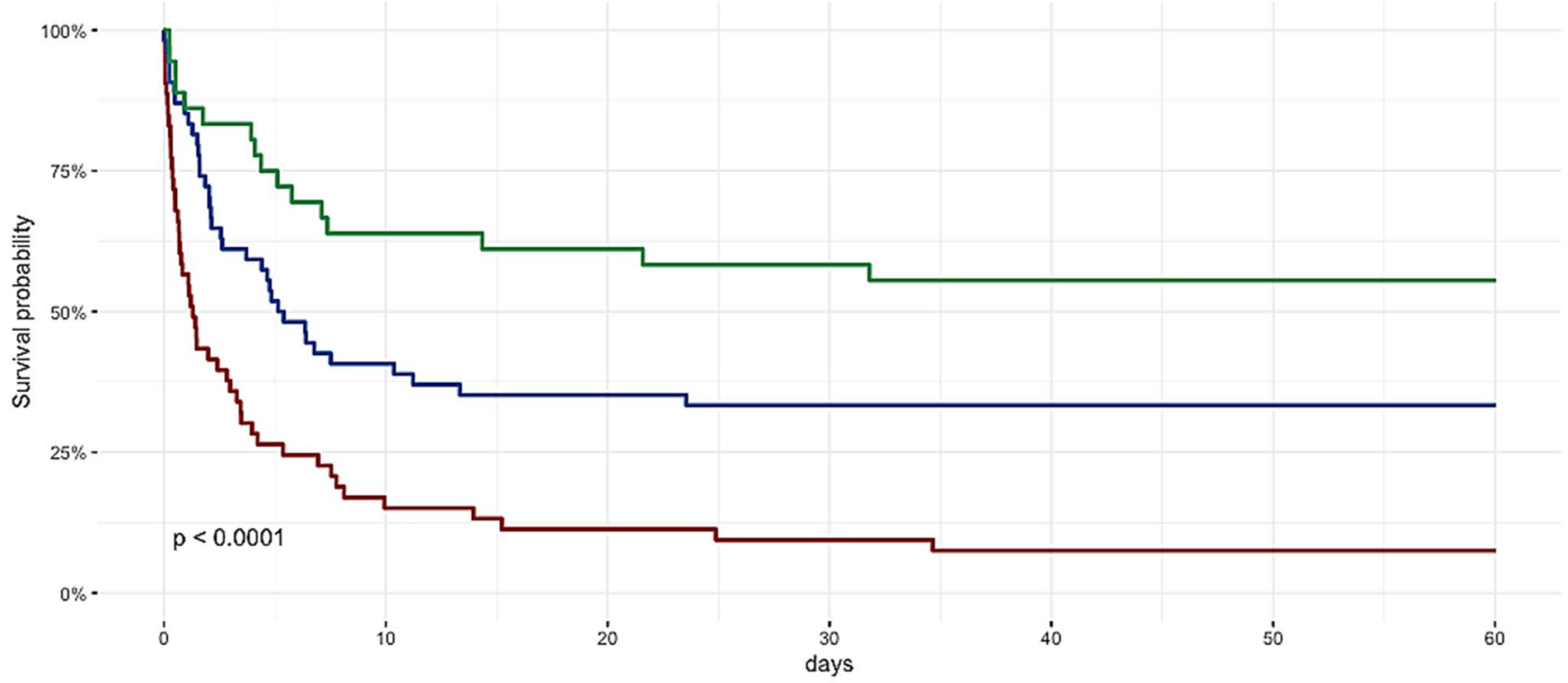

Number at risk

\begin{tabular}{|c|c|c|c|c|c|c|}
\hline$=\quad 53$ & 8 & 6 & 5 & 4 & 4 & 4 \\
\hline$=\quad 54$ & 22 & 19 & 18 & 18 & 18 & 18 \\
\hline$=\quad 36$ & 23 & 22 & 21 & 20 & 20 & 20 \\
\hline 0 & 10 & 20 & $\begin{array}{c}30 \\
\text { days }\end{array}$ & 40 & 50 & 60 \\
\hline
\end{tabular}

Fig. 3 Hospital survival of eCPR patients divided by mean 24 h pulse pressure. Log Rank test: $p<0.001 . e C P R$ extracorporeal cardiopulmonary resuscitation and $P P$ pulse pressure

Survival rates of PP groups in patients without additional mechanical circulatory support (Impella ${ }^{\circledR}$ or IABP) were $6.4 \%$, $31.4 \%$ and $55.6 \%$, respectively $(p<0.001)$.

\section{Analysis of pulse pressure as an independent predictor for survival}

Univariate analysis showed a strong association of high mean $\mathrm{PP}$ of the first $24 \mathrm{~h}$, high mean and systolic arterial pressure as well as a higher level of $\mathrm{pH}$ and standard bicarbonate, a lower level of potassium, glucose and lactate $24 \mathrm{~h}$ after eCPR with increased survival (additional file 1, table E5). Interestingly, diastolic arterial pressure was not associated with survival.

Multivariable analysis revealed mean PP of the first $24 \mathrm{~h}$ after eCPR [OR $3.2(1.3-7.4), p=0.008$ ] and lactate [OR 0.6 $(0.4-0.8), p=0.003]$ as independent predictor for survival (Table 4).
Table 4 Multivariate prognostic analysis $24 \mathrm{~h}$ after eCPR

\begin{tabular}{lll}
\hline & OR $(95 \% \mathrm{CI})$ & $p$ value \\
\hline PP mean of the first 24 h (mmHg) & $3.2(1.3-7.4)$ & $\mathbf{0 . 0 0 8}$ \\
$\mathrm{pH}$ & $3.0(0$ to $>100)$ & 0.795 \\
Lactate $(\mathrm{mmol} / \mathrm{l})$ & $0.6(0.4-0.8)$ & $\mathbf{0 . 0 0 3}$ \\
Standard bicarbonate $(\mathrm{mmol} / \mathrm{l})$ & $0.9(0.7-1.3)$ & 0.623 \\
Potassium $(\mathrm{mmol} / \mathrm{l})$ & $0.4(0.2-1.3)$ & 0.141 \\
Glucose $(\mathrm{mg} / \mathrm{dl})$ & $1.0(1.0-1.0)$ & 0.236 \\
\hline
\end{tabular}

$p$ values $<0.05$ are presented in bold.

Table shows results of a multivariate analysis of the mean PP of the first $24 \mathrm{~h}$ and parameters of blood gas analysis $24 \mathrm{~h}$ after eCPR which were associated with increased or reduced hospital survival in a univariate prognostic analysis

$e C P R$ extracorporeal cardiopulmonary resuscitation and $P P$ pulse pressure 


\section{Discussion}

To our best knowledge, this is the first comprehensive analysis of PP and its meaning for the clinical course of eCPR patients. In this analysis low PP after eCPR was strongly associated with a reduced survival rate. The level of mean PP of the first $24 \mathrm{~h}$ corresponded to a survival rate of $6 \%, 32 \%$ and $56 \%$, respectively. The discrimination between these three groups was present from the first hours after eCPR and lasted up to day 5 .

The patients in this study showed similar baseline characteristics compared to previous reports from eCPR cohorts. This is especially true for important prognostic factors like low flow time, shockable heart rhythm and coronary genesis of cardiac arrest $[10,11,15]$. In addition, this analysis included patients with IHCA, OHCA and pre hospital VA ECMO cannulation, which covers the entire spectrum of eCPR applications. Furthermore, this cohort showed a rather high survival and VA ECMO weaning rate.

The allocation into a low (0-9 $\mathrm{mmHg})$, mid $(10-29 \mathrm{mmHg})$ and high $(\geq 30 \mathrm{mmHg})$ PP group for each time point was based on clinical considerations and resulted in three risk groups. Patients with a low PP showed a survival rate of approx. 5-20\% for each time point and patients with mid and high PP showed a survival rate of approx. $20-40 \%$ and $50-70 \%$, respectively. Thus, not only an association between a very low PP and a high mortality could be shown, but also an association between a high PP and an above the average high survival rate compared to the overall cohort and other eCPR reports (30-40\% survival rate) [4, 9-11]. In a subgroup analysis excluding patients who died of anoxic brain injury, these group differences were even more pronounced, suggesting a possible influence of PP on the physiology and organ function in these patients.

To extend the isolated observation of PP at each single time point, an analysis of the course of PP of all patients within the first days after eCPR was performed. The Sankey chart analysis reveals that patients do not remain permanently in a PP group, but change between the groups to a relevant extent, especially within the first $24 \mathrm{~h}$. As the survival analysis of the three PP groups at each time point suggests, the rate of patients dying is highest in the low PP group. Furthermore, most of the changes between the groups were completed within the first 4 days, so that afterwards most of the patients already died or showed a recovery of the PP and thus changed into the high PP group.

Both from a clinical point of view and due to the high proportion of patients switching between PP groups within the first $24 \mathrm{~h}$, a detailed analysis of the mean PP of the first $24 \mathrm{~h}$ was performed to examine the three PP groups with regard to their baseline characteristics and outcome.
There were several factors that were associated with a low PP level. First of all, patients with a low PP had a higher rate of OHCA compared to patients with mid and high PP. Corresponding to a higher rate of OHCA, the low PP group also showed an at least numerically higher low flow time (10 min longer than patients with mid and high PP). Moreover, patients with a low PP showed higher levels of creatine kinase and creatine kinase-MB. Therefore, the distribution of the duration of low flow time and creatine kinase might be appropriate to explain the level of PP as an indirect parameter for myocardial damage. On the other hand, PP is also determined by the level of arterial afterload. Therefore, it is important to note, that there were neither differences in the applied dose of the inotropic nor the vasoconstrictive therapy between the three PP groups.

In a few patients $(6,4.2 \%)$ with no opening of the aortic valve after inotropic stimulation or in case of severe pulmonary edema, the left heart decompression device Impella ${ }^{\circledR}$ or IABP were used additionally to the VA ECMO therapy. Even though there was no significant difference of left ventricular venting between the groups, this additional support may have led to a further reduced PP. These patients certainly represent a special cohort with an increased risk of mortality, but it seems reasonable to include these patients in this study as well, as it corresponds to real life experience and therapy. Moreover, even after excluding these patients from the analysis, the same association between PP and survival was observed.

Interestingly, there were no differences in no flow time, cause of cardiac arrest or underlying heart rhythm between the three groups. And paradoxically, there was a significantly higher rate of underlying coronary artery disease and a trend for a higher rate of chronic renal failure in patients from the high PP group, which might be explained by the higher rate of IHCA, as the patients were already hospitalised with corresponding diagnoses.

Beyond the extent of a strong association between the PP level and patient outcomes, the mean $24 \mathrm{~h}$ PP appeared as an independent predictor for survival besides the lactate level in a multivariable analysis of parameters available at bedside (vital parameters and blood gas analysis).

The results of this work thus represent a continuation of the findings of previous studies that examined the PP at the time before, during or immediately after VA ECMO cannulation.

Previously, it could be shown that PP before VA ECMO cannulation is an important prognostic parameter in patients with a cardiogenic shock. The survival after veno-arterial ECMO (SAVE) score includes a PP of $\leq 20 \mathrm{mmHg}$ as a factor for increased in mortality [17]. Appropriately, the ECPR score by Park et al. included an initial PP immediately after VA ECMO cannulation of $\geq 24 \mathrm{mmHg}$ as a factor for a higher survival rate [29]. These results are confirmed by a 
study of Ryu et al. in which an initial pulse pressure after eCPR of less than $25 \mathrm{mmHg}$ was associated with inferior neurological outcome [30].

The only study that investigated the influence of the PP in the further course after eCPR, examined the period of the first $6 \mathrm{~h}$ after VA ECMO cannulation. In this mixed cohort (cardiogenic shock, respiratory failure and septic shock) of 69 patients, a high mean PP ( $\geq 30 \mathrm{mmHg})$ was associated with a superior survival and VA ECMO weaning rate [31].

The presented results show for the first time that the prognostic value of the PP is not only present at the time immediately before and after eCPR, but also at any other time within the next 5 days. In summary, we believe that the PP after eCPR, a parameter that is easy to assess at any time, has a high predictive value for survival. Although the PP should not be considered on its own and the shown results should be confirmed in larger trials, it certainly may help in clinical decisions making.

\section{Limitations}

This is a retrospective observational study and, therefore, contains the risk of selection and reporting bias.

Obviously, PP is not only driven by the patient's capability to produce a PP but depends on medical or device treatment. In this analysis inotropic support was similar between the groups and only very few venting devices were used. For that, these confounders should be addressed adequately. However, the present observations can be transferred only to eCPR cohorts with similar treatment regimes.

Therefore, despite of using multivariable analysis to prove PP as an independent predictor for survival, there still might be remaining confounders. Moreover, this is a single-centre report and specific processes may influence the presented results. Together, due to these limitations, our findings about the PP after eCPR should be considered in the overall context of the patient's disease severity and should not prompt therapy limitations on its own.

Furthermore, for the results of the first $12 \mathrm{~h}$ it must be taken into account that due to patient transports and interventions some PP values were missing.

\section{Conclusion}

In this analysis, PP after eCPR was found to be a strong and independent predictor for survival, which could potentially be a useful parameter in daily clinical decision making.

Supplementary Information The online version contains supplementary material available at https://doi.org/10.1007/s00392-021-01838-7.
Author contributions JR and TW contributed to the conception of the study. JR, AR and TW contributed to data collection. JR, AR, XB, MJ, VZ, PMB, DD, KK, CB, DLS and TW contributed to data analysis and interpretation. JR and TW drafted the manuscript. AR, XB, MJ, VZ, PMB, DD, CB, GT, CB and DLS revised the manuscript for important intellectual content. All authors approved the final version of the manuscript.

Funding Open Access funding enabled and organized by Projekt DEAL.. None.

Availability of data and materials The datasets used and/or analysed during the current study are available from the corresponding author on reasonable request.

\section{Declarations}

Conflict of interest The authors declare that they have no competing interests.

Ethics approval and consent to participate The protocol was approved by our institution's ethical committee (EK-Freiburg 151/14).

Open Access This article is licensed under a Creative Commons Attribution 4.0 International License, which permits use, sharing, adaptation, distribution and reproduction in any medium or format, as long as you give appropriate credit to the original author(s) and the source, provide a link to the Creative Commons licence, and indicate if changes were made. The images or other third party material in this article are included in the article's Creative Commons licence, unless indicated otherwise in a credit line to the material. If material is not included in the article's Creative Commons licence and your intended use is not permitted by statutory regulation or exceeds the permitted use, you will need to obtain permission directly from the copyright holder. To view a copy of this licence, visit http://creativecommons.org/licenses/by/4.0/.

\section{References}

1. Go AS, Mozaffarian D, Roger VL, Benjamin EJ, Berry JD, Blaha MJ et al (2014) Executive summary: heart disease and stroke statistics-2014 update: a report from the American Heart Association. Circulation 129:399-410

2. Atwood C, Eisenberg MS, Herlitz J, Rea TD (2005) Incidence of EMS-treated out-of-hospital cardiac arrest in Europe. Resuscitation 67:75-80

3. Soar J, Nolan JP, Bottiger BW, Perkins GD, Lott C, Carli P et al (2015) European resuscitation council guidelines for resuscitation 2015: section 3. Adult advanced life support. Resuscitation 95:100-147

4. Kim SJ, Kim HJ, Lee HY, Ahn HS, Lee SW (2016) Comparing extracorporeal cardiopulmonary resuscitation with conventional cardiopulmonary resuscitation: a meta-analysis. Resuscitation 103:106-116

5. Jacobs I, Nadkarni V, Bahr J, Berg RA, Billi JE, Bossaert L et al (2004) Cardiac arrest and cardiopulmonary resuscitation outcome reports: update and simplification of the Utstein templates for resuscitation registries: a statement for healthcare professionals from a task force of the international liaison committee on resuscitation (American Heart Association, European Resuscitation Council, Australian Resuscitation Council, New Zealand 
Resuscitation Council, Heart and Stroke Foundation of Canada, InterAmerican Heart Foundation, Resuscitation Councils of Southern Africa). Circulation 110:3385-3397

6. Michels G, Wengenmayer T, Hagl C, Dohmen C, Bottiger BW, Bauersachs J et al (2019) Recommendations for extracorporeal cardiopulmonary resuscitation (eCPR): consensus statement of DGIIN, DGK, DGTHG, DGfK, DGNI, DGAI, DIVI and GRC. Clin Res Cardiol. 108:455-464

7. Extracorporeal Life Support Organization (ELSO) (2020): International summary of ECLS registry report, January 2020. https:// www.google.com/url? $\mathrm{sa}=\mathrm{t} \& \mathrm{rct}=\mathrm{j} \& \mathrm{q}=\& \mathrm{esrc}=\mathrm{s} \&$ source $=\mathrm{web} \&$ $\mathrm{cd}=\& \mathrm{cad}=\mathrm{rja} \&$ uact $=8 \& \mathrm{ved}=2 \mathrm{ahUKEwi7oO}$ IttfrAhXBy6QK HRiNDOAQFjAGegQIAxAB\&url=https $\% 3 \mathrm{~A} \% 2 \mathrm{~F} \% 2 \mathrm{Fwww}$. elso.org\%2FPortals\%2F0\%2FFiles\%2FReports\%2F2020_Janua ry\%2FInternational\%2520Summary\%2520January\%25202020 page1.pdf\&usg=AOvVaw2rWvOUPpcX92qGjjCH-8f8. Accessed 07 Sep 2020

8. Stretch R, Sauer CM, Yuh DD, Bonde P (2014) National trends in the utilization of short-term mechanical circulatory support: incidence, outcomes, and cost analysis. J Am CollCardiol 64:1407-1415

9. Shin TG, Choi J-H, Jo IJ, Sim MS, Song HG, Jeong YK et al (2011) Extracorporeal cardiopulmonary resuscitation in patients with inhospital cardiac arrest: a comparison with conventional cardiopulmonary resuscitation. Crit Care Med 39:1-7

10. Kuroki N, Abe D, Iwama T, Suzuki K, Sugiyama K, Akashi A et al (2017) Association between delay to coronary reperfusion and outcome in patients with acute coronary syndrome undergoing extracorporeal cardiopulmonary resuscitation. Resuscitation 114:1-6

11. Wang CH, Chou NK, Becker LB, Lin JW, Yu HY, Chi NH et al (2014) Improved outcome of extracorporeal cardiopulmonary resuscitation for out-of-hospital cardiac arrest-a comparison with that for extracorporeal rescue for in-hospital cardiac arrest. Resuscitation 85:1219-1224

12. Chen YS, Lin JW, Yu HY, Ko WJ, Jerng JS, Chang WT et al (2008) Cardiopulmonary resuscitation with assisted extracorporeal life-support versus conventional cardiopulmonary resuscitation in adults with in-hospital cardiac arrest: an observational study and propensity analysis. Lancet 372:554-561

13. Reynolds JC, Frisch A, Rittenberger JC, Callaway CW (2013) Duration of resuscitation efforts and functional outcome after out-of-hospital cardiac arrest: when should we change to novel therapies? Circulation 128:2488-2494

14. Ouweneel DM, Schotborgh JV, Limpens J, Sjauw KD, Engstrom AE, Lagrand WK et al (2016) Extracorporeal life support during cardiac arrest and cardiogenic shock: a systematic review and meta-analysis. Intensiv Care Med 42:1922-1934

15. Wengenmayer T, Rombach S, Ramshorn F, Biever P, Bode C, Duerschmied D et al (2017) Influence of low-flow time on survival after extracorporeal cardiopulmonary resuscitation (eCPR). Crit Care 21:157

16. Debaty G, Babaz V, Durand M, Gaide-Chevronnay L, Fournel E, Blancher M et al (2017) Prognostic factors for extracorporeal cardiopulmonary resuscitation recipients following out-of-hospital refractory cardiac arrest. a systematic review and meta-analysis. Resuscitation 112:1-10

17. Schmidt M, Burrell A, Roberts L, Bailey M, Sheldrake J, Rycus PT et al (2015) Predicting survival after ECMO for refractory cardiogenic shock: the survival after veno-arterial-ECMO (SAVE)score. Eur Heart J 36:2246-2256
18. Wengenmayer T, Duerschmied D, Graf E, Chiabudini M, Benk C, Muhlschlegel S et al (2019) Development and validation of a prognostic model for survival in patients treated with venoarterial extracorporeal membrane oxygenation: the PREDICT VA-ECMO score. Eur Heart J Acute Cardiovasc Care 8:350-359

19. Stevenson LW, Perloff JK (1989) The limited reliability of physical signs for estimating hemodynamics in chronic heart failure. JAMA 261:884-888

20. Harbaoui B, Nanchen D, Lantelme P, Gencer B, Heg D, Klingenberg R et al (2018) Prognostic value of pulse pressure after an acute coronary syndrome. Atherosclerosis 277:219-226

21 Mitchell GF, Moye LA, Braunwald E, Rouleau JL, Bernstein V, Geltman EM et al (1997) Sphygmomanometrically determined pulse pressure is a powerful independent predictor of recurrent events after myocardial infarction in patients with impaired left ventricular function. SAVE Investigators. Survival and Ventricular Enlargement. Circulation 96:4254-4260

22. Nikolov NM, Fontes ML, White WD, Aronson S, Bar-Yosef S, Gaca JG et al (2010) Pulse pressure and long-term survival after coronary artery bypass graft surgery. AnesthAnalg 110:335-340

23. Aronson D, Burger AJ (2004) Relation between pulse pressure and survival in patients with decompensated heart failure. Am J Cardiol 93:785-788

24. Mourad M, Eliet J, Zeroual N, Saour M, Sentenac P, Manna F et al (2020) Pulse pressure and end-tidal carbon dioxide for monitoring low native cardiac output during veno-arterial ECLS: a prospective observational study. Crit Care 24:569

25. Le Gall JR, Lemeshow S, Saulnier F (1993) A new simplified acute physiology score (SAPS II) based on a European/North American Multicenter Study. JAMA 270:2957-2963

26 Bemtgen X, Heidt T, Zotzmann V, Rilinger J, Wengenmayer T, Biever PM et al (2020) Venoarterial extracorporeal membrane oxygenation decannulation using the novel Manta vascular closure device. Eur Heart J Acute Cardiovasc Care. https://doi.org/10. 1177/2048872620918707

27. Staudacher DL, Gold W, Biever PM, Bode C, Wengenmayer T (2017) Early fluid resuscitation and volume therapy in venoarterial extracorporeal membrane oxygenation. J Crit Care 37:130-135

28. Zotzmann V, Rilinger J, Lang CN, Duerschmied D, Benk C, Bode C et al (2019) Early full-body computed tomography in patients after extracorporeal cardiopulmonary resuscitation (eCPR). Resuscitation. https://doi.org/10.1016/j.resuscitation.2019.11.024

29. Park SB, Yang JH, Park TK, Cho YH, Sung K, Chung CR et al (2014) Developing a risk prediction model for survival to discharge in cardiac arrest patients who undergo extracorporeal membrane oxygenation. Int J Cardiol 177:1031-1035

30. Ryu JA, Chung CR, Cho YH, Sung K, Jeon K, Suh GY et al (2019) Neurologic outcomes in patients who undergo extracorporeal cardiopulmonary resuscitation. Ann ThoracSurg 108:749-755

31. Park BW, Seo DC, Moon IK, Chung JW, Bang DW, Hyon MS et al (2013) Pulse pressure as a prognostic marker in patients receiving extracorporeal life support. Resuscitation 84:1404-1408

32. R Core Team (2014). R: A language and environment for statistical computing. R Foundation for Statistical Computing, Vienna, Austria. http://www.R-project.org/.

33. Wickham H (2016). ggplot2: Elegant Graphics for Data Analysis. Springer-Verlag New York. ISBN 978-3-319-24277-4 